\title{
Quantifying Learning Creativity through Simulation and Modeling of Swarm Intelligence and Neural Networks
}

\author{
doi:10.3991/ijoe.v7i2.1642 \\ Hasan M. Mustafa1 ${ }^{1}$, Turki F. Al-Somani ${ }^{1}$, Ayoub Al-Hamadi ${ }^{2}$ \\ 1 Al-Baha University, Al-Baha, Kingdom of Saudi Arabia \\ ${ }^{2}$ Otto-von-Guericke-University Magdeburg, Germany
}

\begin{abstract}
This research work presents a systematic investigational study of a challenging phenomenon observed in natural world. Mainly, the study is concerned with conceptual interdisciplinary analysis and evaluation of quantified learning creativity phenomenon. In association, it deals with diverse aspects of measurable behavioral learning performance and is observed by two diverse natural biological system models (i.e. human and non-human creatures). Specifically, the studies of two biological models consider the comparison of quantified learning creativity phenomenon. The first model involves the human interactive tutoring/learning processes with environment while the other modal presents the ecological behavioral learning of swarm intelligence agents (i.e. ants) in performing the foraging process. Furthermore, a comparative study is presented which is inspired by naturally realistic models of Artificial Neural Network (ANN) and Swarm Intelligence. The obtained simulation and modeling results shows that the learning performance curves of both models behave with close similarity to each other. Precisely, the analysis and evaluation of learning performance curves of two diverse biological models revealed that both obey exponentially decayed learning curves; following least mean square (LMS) error algorithm.
\end{abstract}

Index Terms-Learning creativity phenomenon, Synaptic Plasticity, Artificial Neural Network Modeling, Learning Creativity, Ant Colony Systems, and Computational Biology.

\section{INTRODUCTION}

This piece of research is inspired by a strong belief that interdisciplinary combination of Neural Networks models with cognitive learning theory and neuroscience contributes innovative investigations of essential educational issues. Specifically, systematic investigational study of quantified human learning creativity phenomenon is considered as an interdisciplinary, challenging, and interesting educational issue. Moreover, human creativity phenomenon is observable while practicing interactive face to face tutoring sessions at classrooms. In other words, learning creativity phenomenon is detectable at educational field practice, during performing mutual bidirectional feedback (i.e. input stimulation/output response) between tutor and learner.

This paper adopts realistic simulation and modeling of two relatively new creativity disciplines concerned with (i.e. humans and non-humans). Accordingly, it presents an investigational comparative study of observed learning creativity phenomenon associated with both creative disciplines. They are named as: swarm intelligence and neural networks which are modeled realistically aiming to reach innovative quantitative investigational study of observed creature's creativity phenomenon in nature. The presented creativity models in this work are tightly related with behavioral learning convergence observed by humans (i.e. neural) and Ant Colony System (i.e. non neural) $[1,2]$.

In the first modal, Artificial Neural Networks (ANN ${ }^{\mathrm{S}}$ ) modelling discipline has been adopted for realistic modeling of quantified human learning creativity phenomenon. So, the presented $\mathrm{ANN}^{\mathrm{S}}$ models have been designed and implemented on the bases of optimal selectivity of two ANN ${ }^{\mathrm{S}}$ design parameters. Namely: gain factor of Sigmoid activation function and learning rate parameter value. Furthermore, optimal choice of number of hidden neurons is necessary for enhancement of quantified learning creativity. Conclusively, presented simulation and modeling results for either learning paradigms seemed to be promising for more elaborative future, systematic and innovative applied research in evaluation and enhancement of human learning creativity phenomenon [3-6]. In the second model, ecological behavioral learning of Ant Colony System (ACS) is studied having the name: Temnothorax albipennis (formerly Leptothorax albipennis). Its individual agents (i.e. ants) adopt the natural intelligent teaching technique known as tandem running.

Briefly, the type of ACS adopting (i.e. tandem running technique) performs its behavioral learning function sequentially as follows. In case of one ant running to lead another ant while moving from the nest to food, both leader and follower (teacher and pupil) are acutely adaptive sensitively to the progress of their partner. To the best of our knowledge; agents of Leptothorax albipennis ACS perform a creative inter-communication technique among ants. Moreover, it involves teaching by interactive feedback between two ants controlling trade-off between speed and accuracy $[2,7,8]$.

The rest of this paper is composed of five sections and are organized as follows. In the next section, a brief overview is given for the basic human learning. An example of human learning creativity is presented in the third section. In the fourth section, an example is introduced for learning creativity concerned with one type of Ant Colony System adopting learning by tandem running technique. It is named as: Temnothorax albipennis (formerly Leptothorax albipennis). The simulation results for both creature 
types (human and non human) are shown in fifth section. The last section illustrates some interesting conclusive remarks and suggestions for future work. Finally, three appendices are presented at the end of this paper to illustrate the results after running a computer program, and Computer Assisted Learning Package.

\section{BASIC HUMAN LEARNING MODEL}

From neuro-physiological point of view, practical performance of learning process utilizes two essential cognitive brain functions. Both of these functions are needed for efficiently performing learning/tutoring interactive process in accordance with behaviourism [9-11] as follows. Firstly, pattern classification/recognition creativity function based on visual/audible interactive neuronal signals via sensory organs (eyes/ears). Consider the ANN ${ }^{\mathrm{S}}$ modelling which recognizes brain function performed originally via neuronal signal's perception. Essentially, this function needs a supervisor's intervention to reach learning completion (convergence). So it is modelled by adopting principle of learning (under supervision) paradigm [12]. Secondly, associative memory brain function, which is based on Hebbian learning rule and motivated originally by classical conditioning (Pavlovian) learning. It belongs to the principle of learning without a teacher (unsupervised) [13]. In other words, in the context of educational practice, learning processes performed either by interaction with a teacher (face to face learning) or via computer aided learning software.

Figure 1 illustrates the teaching model in performing realistic simulation of the two mentioned brain functions. Inputs to the adopted learning creativity model in Figure 1 are provided by environmental stimuli (unsupervised learning).The correction signal for the case of learning with a teacher is given by responses outputs of the model and will be evaluated either by the environmental conditions (unsupervised learning) or by the teacher. Finally, the tutor plays a role in improving the input data (stimulating learning pattern) by reducing noise and redundancy of model pattern input. It is according to tutor's experience to provide the model with clear data by maximizing its signal to noise ratio. However, our case is based on unsupervised Hebbian rule, which is originated from self-organized (autonomous) learning, [13].

\section{EXAMPLE OF HUMAN LEARNING CREATIVITY}

Human learning creativity issue essentially associated with educational performance assessment considers the learning and memory brain functions. Consequently, the

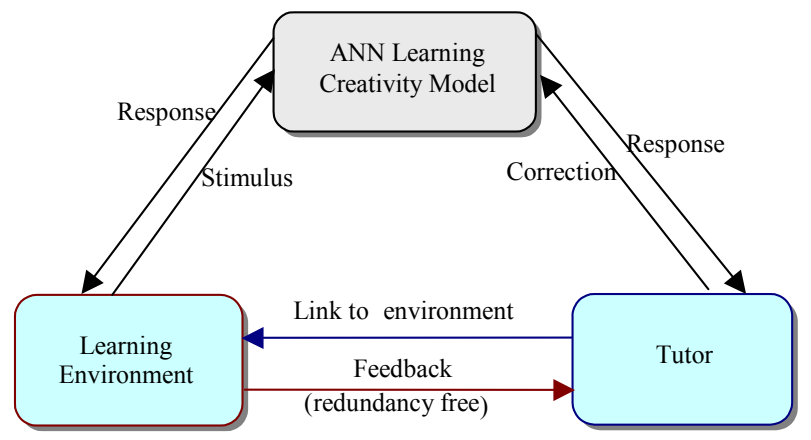

Figure 1. Schematic diagram for an interactive learning/tutoring process. presented example in this section is motivated by cognitive theory as an optimal approach for improving teaching/ learning performance of a mathematical topic of children at the fifth grade (in elementary schools). The optimal cognitive approach is supported by National Institutes of Health (NIH) in U.S.A. by considering children at the fifth grade class level. They are about 11 years old at the age of elementary schools' at which children may be qualified to learn "basic building blocks" of cognition [14]. Moreover, a new research which shows the prefrontal cortex handles the work of associating numerals with matching quantities [15]. Both of these research activities have motivated our approach for modelling mathematical creativity using ANNs.

This paper aims to discovery of children creativity in mathematics (of about 11years age) and for that, a specific mathematical topic is suggested which depends on algorithmic solution of long division problems following the sequential mathematical steps: Divide, Multiply, Subtract, Bring Down, and repeat if necessary (See algorithm presented in APPENDIX I). Accordingly, the given example adopts creativity assessment issues and is concerned with cognitive multimedia theory application at educational field practice (classrooms) [16-19]. Based on this theory; visual and auditory material should be presented simultaneously to reinforce retention of a learnt mathematical topic [18-20]. A sample of the print screen of designed package is given in APPENDIX II which presents discovery of mathematical creativity.

\section{AN EXAMPLE OF ACS CREATIVITY}

\section{A. Tandem running technique}

Referring to Figure 2, it illustrates schematically the learning paradigm inspired from tandem running technique and is adopted by an ACS of type namely: Temnothorax albipennis (formerly Leptothorax albipennis). This technique involves interactive bidirectional feedback between teacher and pupil corresponding to leader and follower ants respectively. Furthermore, in this figure, depicted block named as (Follower/Leader) suggests that tandem follows after learning their lessons so well, that they can become tandem leaders.

In co-operative learning context, the previous type of ACS behavioral learning performance [21, 22] is analog and can be observed in classrooms; if one agent (student) behaves independently from other agent's achievements [23]. So, it is described as teacher-centered providing Individual learning which implies that leader ant (teacher) can transfer knowledge and cognitive skill to the learner (another ant) $[23,24]$. Accordingly, via that teacher-

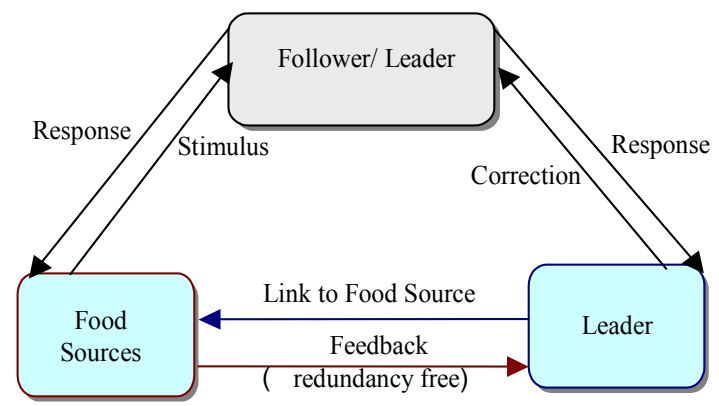

Figure 2. Schematic diagram for a tandem running technique with bidirectional feedback. 
centered type of learning, the teacher provides the major source of information and feedback [25]. Conclusively, ANN models based on supervised learning paradigm are relevant for realistic simulation of cooperative teachercentered learning performance $[23,24]$.

\section{B. Solution of Travelling Salesman Problem by ACS Algorithms}

Another type of ACS which is capable to converge the optimal solution of Traveling Salesman Problem (TSP) is by adopting an autocatalytic ACS algorithm. Moreover, the optimal solutions of TSP are obtained by various speeds in accordance with inter-communication levels among ACS agents [26, 27]. Referring to Figure 3, qualitative performance of autocatalytic ACS algorithm is illustrated for different inter-communication levels among ants. The dashed curve denotes qualitatively lower intercommunication level than the continuous one. Moreover, convergence to optimal TSP solution can easily be reached by increasing the computer CPU time [28]. Realistically, this time corresponds to stored experience among ACS agents which may results in optimal spontaneous response to solve TSP. By following another ANT-density algorithm, the same ACS type is capable of solving TSP optimally. The simulation results of its application are illustrated at Figure 4. Referring to [29], it shows that efficiency per ant (required to reach optimal TSP solution) is well improved as number of ants increase. Furthermore, it is observed that number of trials increase at Thorndike's psycho-learning experimental model and is analogous to number of ants at ANT-density algorithm [7]. Finally, the presented model seems to take into account the mixed learning paradigms constituted from the reinforcement learning [30], correction of back-propagation error [12], and learning by interaction with environmental conditions [31].

It is to be noted that the graphs in figure 3, are closely similar to each other and agree with the least mean square (LMS) error algorithm curves following different learning conditions parameters such as learning rate values and are analogous to different inter-communication levels among ants $[7,26]$.
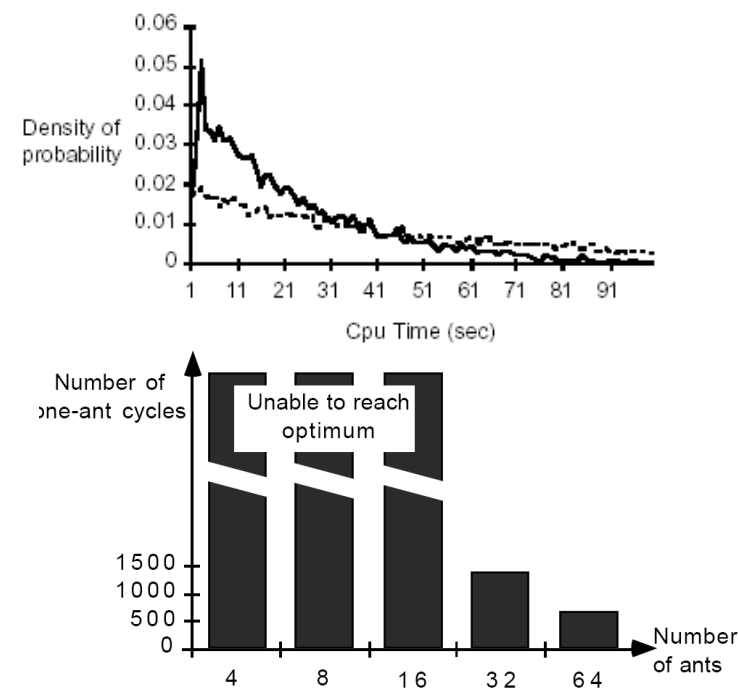

Figure 3. Top: PDF of finishing CPU times to reach optimal TSP problem solution for different inter-communication levels [26]. Button: Number of cycles required to reach optimum rated to the total number of ants [29].

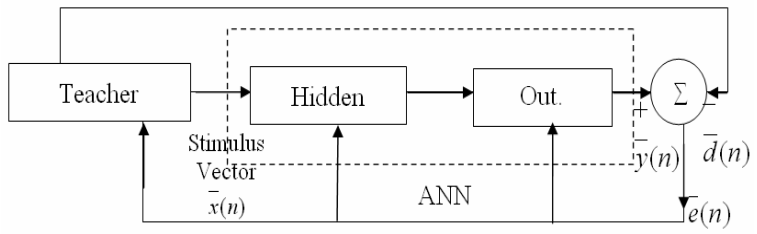

Figure 4. Block diagram for suggested ANN model for error correcting learning paradigm, adapted from [20].

\section{Analogy between ANNs and ACS}

According to presented analysis of the learning behaviour of the Leptothorax albipennis ACS in the previous subsection $(A)$, figure 4 presents a relevant ANN model which simulates realistically ACS learning processes by considering supervised (guided-error correction) algorithm (equivalently: learning with a teacher). Moreover, it is analogous to (Follower/Leader) performance according to adopted tandem running technique based on paradigmatic decentralized decision-making [8,21].

The observed error vector $\bar{e}(n)$ at any time step $n$ during the learning processes is given by:

$$
\bar{e}(n)=\bar{y}(n)-\bar{d}(n)
$$

Where $\bar{y}(n)$ is the output is signal of the model and $\bar{d}(n)$ is the numeric value(s) of the desired/objective parameter of learning process. Referring to figure 4, the following equations are considered:

$$
\begin{gathered}
V_{k}(n)=X_{k}(n) \cdot W_{k j}^{T}(n) \\
y_{k}(n)=\varphi\left(V_{k}(n)\right)=\left(1-e^{-\lambda v_{k}(n)}\right) /\left(1+e^{-\lambda v_{k}(n)}\right) \\
e_{k}(n)=\left|d_{k}(n)-y_{k}(n)\right| \\
W_{k j}(n+1)=W_{k j}(n)+\Delta W_{k j}(n)
\end{gathered}
$$

Where $X$ is input vector, $W$ is the synaptic weight vector, and $\varphi($.$) is an activation (odd sigmoid) function asso-$ ciated to the $\mathrm{k}^{\text {th }}$ neuron. It is characterized by its argument $\mathrm{V}_{\mathrm{k}}(\mathrm{n})$, gain factor $\lambda$ and the developed output at the $\mathrm{n}^{\mathrm{th}}$ leaning instant $y_{k}(n)$ is given by equation (3). Furthermore, by comparing the pre-assumed desired output $d_{k}$ with the actual developed output $\mathrm{y}_{\mathrm{k}}$, the error value $e_{\mathrm{k}}$ is obtained by equation (4). It is noticed that $\Delta W_{\mathrm{kj}}(n)$ represents the dynamical change at the $n^{\text {th }}$ leaning instant of synaptic weight vector value connecting the $\mathrm{k}^{\text {th }}$ and $\mathrm{i}^{\mathrm{th}}$ neurons. Moreover, it is worthy to note that the equations (2-5) are commonly valid for two diverse ANN learning paradigms namely; supervised (interactive learning with a tutor), and unsupervised (learning though students' selfstudy). Specifically, in this work supervised (interactive) learning paradigm is considered. So, dynamical changes of weight vector value (connecting the $\mathrm{k}^{\text {th }}$ and $\mathrm{i}^{\mathrm{th}}$ neurons) for supervised learning process is given by following equation:

$$
\Delta W_{k j}(n)=\eta e_{k_{j}} X(n)
$$

Where $\eta$ is the learning rate value while performing the learning process. 


\section{PAPER: QUANTIFYING LEARNING CREATIVITY THROUGH SIMULATION AND MODELING OF SWARM INTELLIGENCE AND NEURAL NETWORKS}

\section{Simulation Results of Human \& ACS QuANTIFIED CREATIVITY MODELS}

Referring to some published study concerned with creativity in mathematics education [32] and to recently published work [33] which deals with the study of early discovery of mathematical learning creativity. The analysis and evaluation of convergence time is presented using ANN modeling. Additionally, it has been declared that application of improved educational technology methodologies implies virtual enhancement of learning creativity after the increase of learning rate values [6]. Obviously, the enhancement of learning creativity results in better learning performance quality indicated by minimizing the learning convergence (response) time (fig. 5 and 6) and as well minimizing error values (fig. 7). The flowchart is given in APPENDIX III which describes simulation algorithm. The running time of this program results in three set of curves as shown in fig. 5, 6 and 7.

In agreement with all of the above set of curves shown in three figures (fig. 6, 7, and 8), they are all with close similarity to exponentially decayed performance curves. A normalized abstract set of decayed exponential curves are given in figure 7 and is followed by the mathematical formula in which $\eta_{i}$ is defined as learning rate factor. The set of various learning rate factor values are denoted by $\eta_{i}$. These factor values are mathematically presented after normalization of different learning performance curves (at Figure 9) as follows:

$$
y(n)=e^{-\eta_{i}(n-1)}
$$

Where $\mathrm{n}$ is the number of training cycles. Referring to figures 7 and 8, the effect of gain factor as well as learning rate changes on co-operative learning performance is introduced. It is interesting to note that analysis of introduced effect is supported well by the exponentially decayed graphs described by equation (7) and illustrated set of curves at Figure 8.

\section{CONCLUSION AND DISCUSSION}

Five conclusive remarks can be made: Quantitative evaluation of learning creativity is well promising to improve learning performance quality as measured after investigational analysis of obtained educational field results [6].

Early discovery of children mathematical creativity could be attained via presented assessments of children response time after solving long division problem [32-33, 19]. A simplified macro level flowchart describes the basic algorithmic steps considered is presented at APPENDIX I for solving suggested Long Division problem. Furthermore, samples of output print screen are shown in APPENDIX II.

The presented comparison study of two learning creativity models associated with human and non-human creatures has revealed their mutual analogy between each other. Both obey exponentially decayed learning curves; following least mean square (LMS) error algorithm [12] that is described by equation (7) and illustrated by the set of curves in Figure 9.

Increasing number of cognitive sensory neurons (hidden neurons) participating in tutoring processes results in better development of learning creativity and more addi-

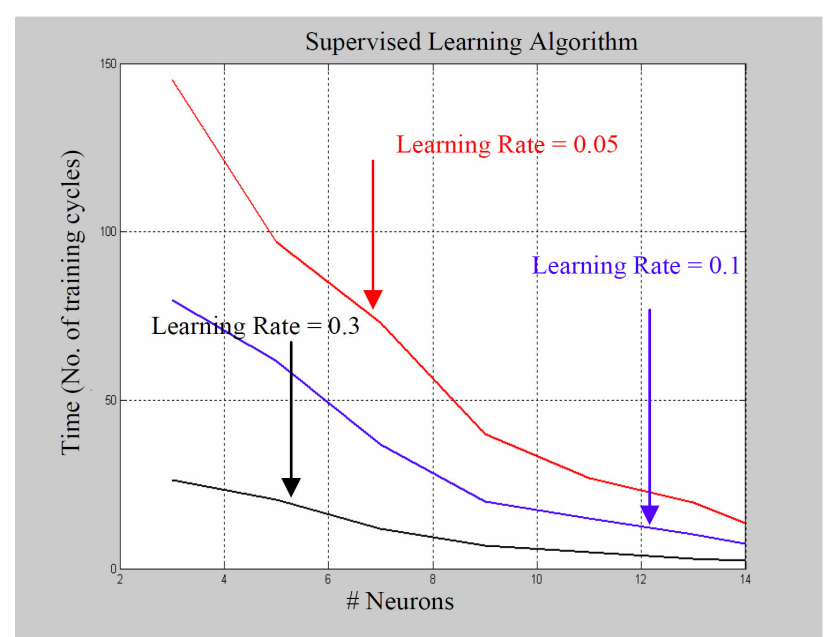

Figure 5. Error correction performance based on time response parameter with considering three different learning rates: $0.05,0.1$ and 0.3 for gain factor $=0.5$, while \#cycles $=300$.

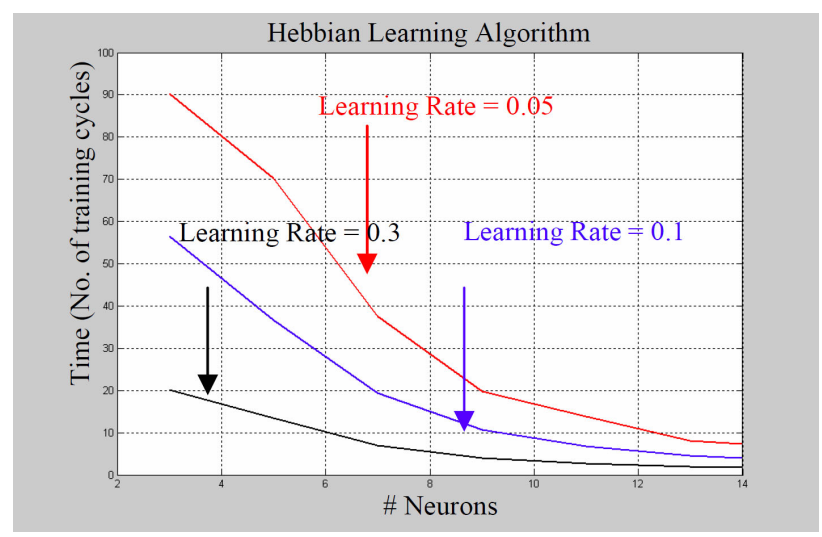

Figure 6. Hebbian learning performance and time factor with considering three different learning rates: $0.05,0.1$ and 0.3 for gain factor $=0.5$, while \#cycles $=300$.

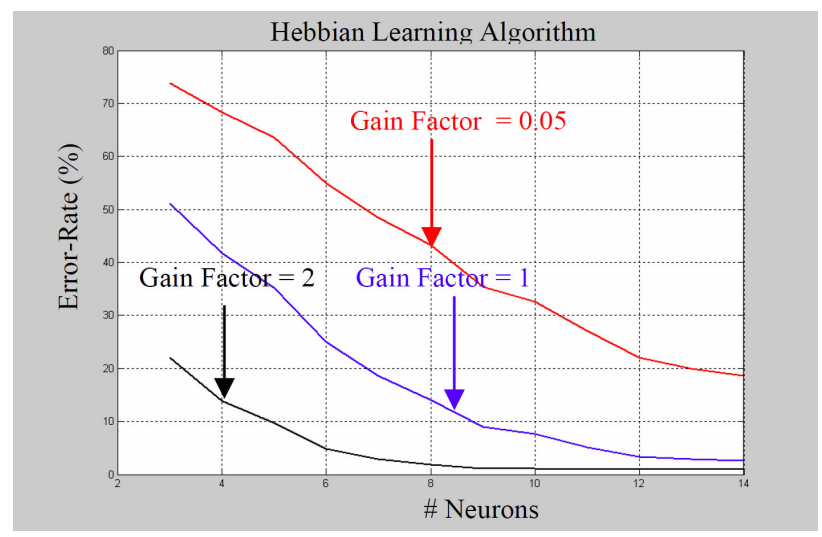

Figure 7. Hebbian learning performance error-rate with different gain factors : 0.05 , 1, and 2, while \#cycles $=300$ and Learning rate $=0.3$.

tive value for educational performance quality. This remark is well supported by obtained results shown in figures $(6,7$, and 8$)$ and by referring APPENDIX III.

The stored experience during Hebbian process as well as computational intelligence process of ACS; both are analogues to computer CPU time (while running simulation programs) in order to develop error minimization for reaching desired output (optimal solution). 


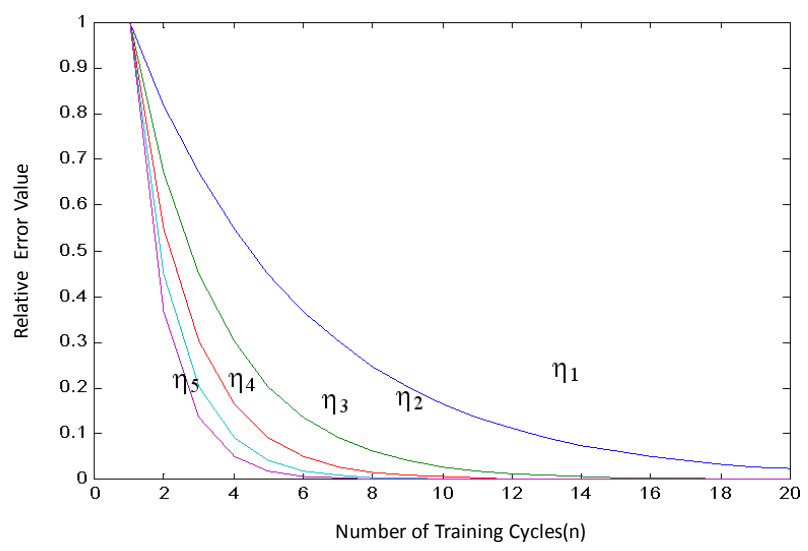

Figure 8. Learning performance curves for different learning rate factor values $\left(\eta_{i}\right)[10]$.

Finally, presented analysis and evaluation are based on suggested ANNs modelling shed light on promising future extension enhancement of learning performance quality by considering interestingly revealed resemblance of quantified learning creativity phenomenon associated with both (human and non-human) creature's types. Additionally, as a consequence of all the remarks, it is worthy to recommend realistic implementation of ANNs models for solving more complex educational phenomena issues related to cognitive styles such as early discovery of learning creativity, quality assurance of learning performance, evaluation of students' diversity learning styles, cooperative learning modeling, Learning under noisy data environment, learning disabilities, etc. [34,35]

\section{REFERENCES}

[1] H.M.Hassan "On Principles of Biological Information Processing Concerned with Learning Convergence Mechanism in Neural and Non-Neural Bio-Systems." Published at IEEE conference, CIMCA 2005 Vienna, Austria.

[2] H.M.Hassan,"A Comparative Analogy Between Swarm Smarts Intelligence and Neural Network Systems.", published at WSSEC08 conference held on 18-22 August 2008, Derry, Northern Ireland

[3] H.M.Hassan "On Quantifying Learning Creativity Using Artificial Neural Networks (A Mathematical Programming Approach)" published at CCCT 2007 conference held on July12-17, 2007, Orlando, Florida, USA.

[4] H.M.Hassan, A.Al-Hamadi, and F.B.Al-Mohaya "On Quantifying Learning Creativity Using Artificial Neural Networks (A Nerophysiological Cognitive Approach)" National Conference on Applied Cognitive Psychology held in India, Calcutta,2007.

[5] H.M.Hassan, "On Analysis of Quantifying Learning Creativity Phenomenon Considering Brain Synaptic Plasticity", published at WSSEC08 conference held on 18-22 August 2008 ,Derry, Northern Ireland.

[6] H.M.Hassan, "On Evaluation of Virtual Improvement of Learning Creativity by Application of Computer Assisted Learning Using Artificial Neural Networks Modeling.", Sixth Annual International Conference on Remote Engineering and Virtual Instrumentation REV2009 2009 at University of Bridgeport, CT, USA.

[7] H.M.Hassan," A Comparative Analogy of Quantified Learning Creativity in Humans Versus Behavioral Learning Performance in Animals: Cats, Dogs, Ants, and Rats.(A Conceptual Overview), published at WSSEC08 conference held on 18-22 August 2008, Derry, Northern Ireland .

[8] N.R.Franks and T.Richardson (2006). Teaching in tandem-running ants. Nature 439 (7073): 153. PMID 16407943. doi: $10.1038 / 439153 \mathrm{a}$

[9] H.M.Hassan and M.Watany, 2000: On Mathematical Analysis of Pavlovian Conditioning Learning Process using Artificial Neural
Network Model", 10th Mediterranean Electro technical Conf., Cyprus.

[10] H.M.Hassan and M.Watany, 2003: On Comparative Evaluation And Analogy For Pavlovian And Throndikian Psycho-Learning Experimental Processes Using Bioinformatics Modelling, AUEJ, 6,3, pp.424-432.

[11] I.P.Pavlov: Conditional Reflex, An Investigation of The Psychological Activity of the Cerebral Cortex, 1927, New York, Oxford University press.

[12] S.Haykin, Neural Networks, Englewood Cliffs, NJ: Prentice-Hall, 1999.

[13] D.O. Hebb, "The organization of behaviour", Wiley, New York (1949).

[14] N.Swaminathan, "Cognitive Ability Mostly Developed Before Adolescence, NIH Study Says. NIH announces preliminary findings from an effort to create a database that charts healthy brain growth and behavior „Scientific American letter, May 18, 2007.

[15] N.Swaminathan, "How the Brain Maps Symbols to Numbers" Scientific American letter, October, 2007.

[16] S.Tindall-Ford, P.Chandler, and J.Sweller, "When Two Sensory Modes are Better than One", Journal of Experimental Psychology: Applied, Vol. 3, 1997, pp.257-287. doi:10.1037/1076-898X.3.4 . $\underline{257}$

[17] R.Moreno and R.E.Mayer, "Cognitive Principles of Multimedia Learning: The Role of Modality and Contiguity", Journal of Educational Psychology, Vol. 91,1999, pp. 358-368. doi:10.1037/ 0022-0663.91.2.358

[18] R.Lindstrom, The Business Week Guide to Multimedia Presentations: Create Dynamic Presentations That Inspire, New York: McGraw-Hill, 1994.

[19] H.M.Hasan, A.Al-Hamadi, and M.H.Kortam "On Assessment of Teaching A Mathematical Topic Using Neural Networks Models ( with a case study) ", conference proceeding of Technology and its Integration into Mathematics Education Time 2010 , Malaga Spain, July 6th-10th, 2010.

[20] F.A.Al-Zahrani, H.M.Hasan, and A. Al-Hamadi, "On Analysis And Evaluation of Multi-Sensory Cognitive Learning Of A Mathematical Topic Using Artificial Neural Networks", Journal of Telecommunications, 1(1), 2010, 99-104.

[21] J.A.Marshall, A.Dornhaus, N.R.Franks, T.Kovacs."Noise, cost and speed-accuracy trade-offs: decision-making in a decentralized system" J R Soc Interface. Royal Society Publishing, 2006, Apr 22;3(7):243-54.

[22] S.Garnier, J.Gautrais, and G.Theraulaz" The biological principles of swarm intelligence "Swarm Intelligence Journal Volume 1, Number 1 / June, 2007, pp.3-31

[23] D.Johnson, R.Johnson Learning Together and Alone ed3.; Allyn \& Bacon, Sydney, 2010, pp.2.

[24] PLSB, Professional Learning Services Branch, 1999, Five Basic Elements of Cooperative Learning [Online access14/6/02] URL: http://web.ec.tased.edu.au/las/sose/fiveelem.htm

[25] G.Salomon, D.Perkins, P. Theroux, 2001 Comparing Traditional Teaching and Student Centered, Collaborative Learning Online, accessed14/6/02]URL: http://shaw.ca/priscillatheroux/collaborati ve.html

[26] M.Dorigo, Ant colonies for the travelling salesman problem, A set of papers at a web site http://www.iridia.ulb.ac.be/ dorigo/aco/aco.html ,1997.

[27] E.Bonabeau, and G.Theraulaz, "Swarm Smarts" Majallat Aloloom, May 2001, vol 17, No 5, pp 4-12.

[28] M.A.Ghonaimy, N.A.Raslan, and H.M. Hassan, "Dynamic Modeling of Multiprocessor Controlled Exchanges " FOURTH NATIONAL RADIO SCIENCE SYMPOSIUM, (MTC) Cairo , Egypt , November , 9-11-1986

[29] C.Alberto, Distributed optimization by ant colonies. Proceeding of ECAL91, Elsevier Publishing, 2010, pp 134-142.

[30] E.D.Mariarty, Evolvolutionary algorithms for reinforcement learning, Journal of AI research 11, 1999, pp.241-276.

[31] M.Fukaya, Two level Neural Networks: Learning by Interaction with Environment, 1st ICNN, 1988, San Diego.

[32] H.Meissner, "Creativity and Mathematics Education" published at : Proceedings of the International Conference on Creativity in 


\section{PAPER: QUANTIFYING LEARNING CREATIVITY THROUGH SIMULATION AND MODELING OF SWARM INTELLIGENCE AND NEURAL NETWORKS}

Mathematics Education and the Education of Gifted Students MCG2002. Riga, Latvia 2002

[33] H.M.Hasan "On Early Discovery of Mathematically Creative Children Using Artificial Neural Networks Modeling (with a case study)", 6th International conference on "Creativity in Mathematics Education and the Education of Gifted Students" held in Riga, Latvia, on August 2nd-6th, 2010.

[34] M.A.Ghonaimy, A.M.Al-Bassiouni, and H.M.Hassan, "Leaning Of Neural Networks Using Noisy Data". Published at the Second International Conference on Artificial Intelligence Applications, AUC, Cairo, Egypt, Jan 22-24, 1994. PP. 387-399.

[35] M.A.Ghonaimy, A.M.Al-Bassiouni, and H.M.Hassan, "Learning Ability in Neural Network Model", Published at the Second International Conference on Artificial Intelligence Applications, AUC, Cairo, Egypt, Jan. 22-24, 1994, PP-400-413.

\section{AUTHORS}

Hasan M. Mustafa (mustafa_hasan47@yahoo.com) is with the Computer Engineering Department, Faculty of Engineering, Al-Baha University, Al-Baha, Kingdom of Saudi Arabia.

Turki F. Al-Somani (tfsomani@uqu.edu.sa) is with the Computer Engineering Department, Faculty of Engineering, Al-Baha University, Al-Baha, Kingdom of Saudi Arabia.

Ayoub Al-Hamadi (ayoub.al-hamadi@ovgu.de) is with the Institute for Electronics, Signal Processing and Communications (IESK), Otto-von-Guericke-University Magdeburg, Germany.

This article is an extended version of a paper presented at the IEEE EDUCON20211 Conference, held from April 4th-6th, 2011, in Amman, Jordan. Received April $21^{\text {st }}$, 2011. Published as resubmitted by the authors April $29^{\text {th }}, 2011$

\section{APPENDIX I}

The figure shown below illustrates a simplified macro level flowchart which describes briefly basic algorithmic steps considered by suggested Computer Assisted Learning package. It is designed to perform fairly unbiased assessment process of learning a mathematical topic. After the running of the program, children time response (scores) are obtained, (samples of print screens is shown at APPENDIX II). These samples are obtained in accordance with steps of long division process: Divide, Multiply, Subtract, Bring Down, and repeat (if necessary) as given in reference [32].

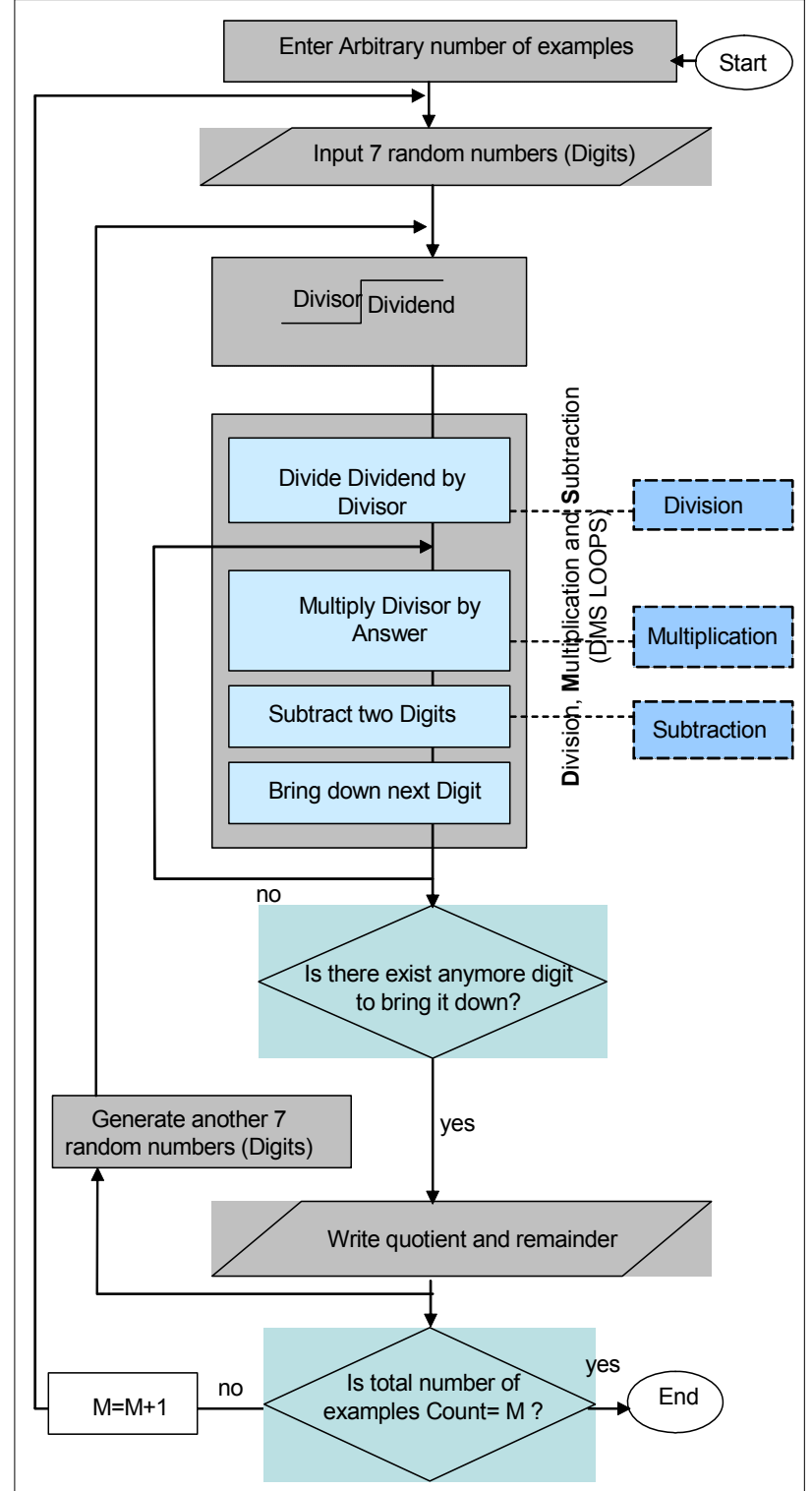

Figure 1. 


\section{PAPER: QUANTIFYING LEARNING CREATIVITY THROUGH SIMULATION AND MODELING OF SWARM INTELLIGENCE AND NEURAL NETWORKS}

\section{APPENDIX II}

Three Print Screen samples are shown in Figures (A, B, and $\mathrm{C}$ ) to illustrate three different output phases of mathematical creativity assessment package.

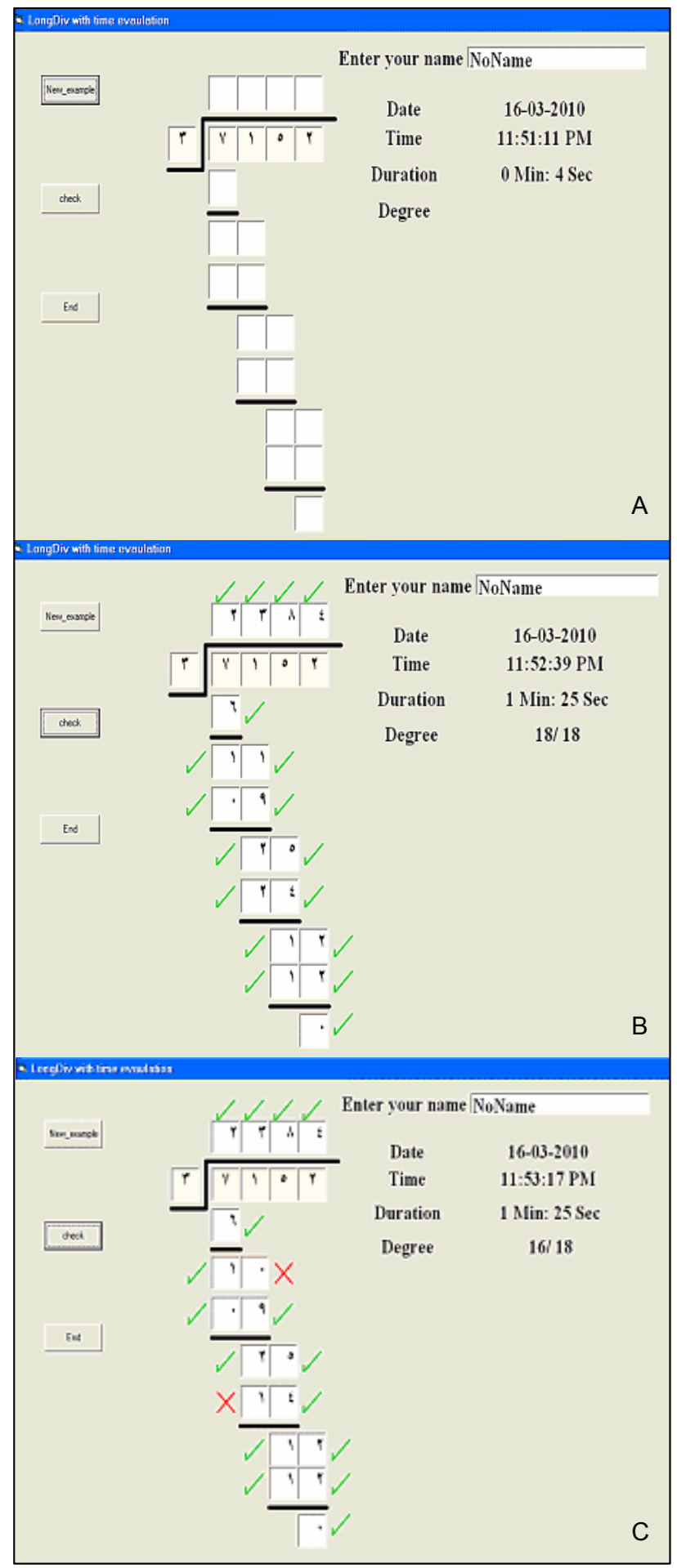

Figure 2. A) Basic print screen sample for initial mathematical Long Division process. B) For fairly solving of Long Division problem (detecting no mistake). C) A print screen for fairly assessment processes results with two mistakes.

\section{APPENDIX III}

The shown simplified macro-level flowchart in below briefly describes algorithmic steps for realistic simulation learning program using Artificial Neural Networks. The results are shown in three figures $(6,7$, and 8$)$ after running the program.

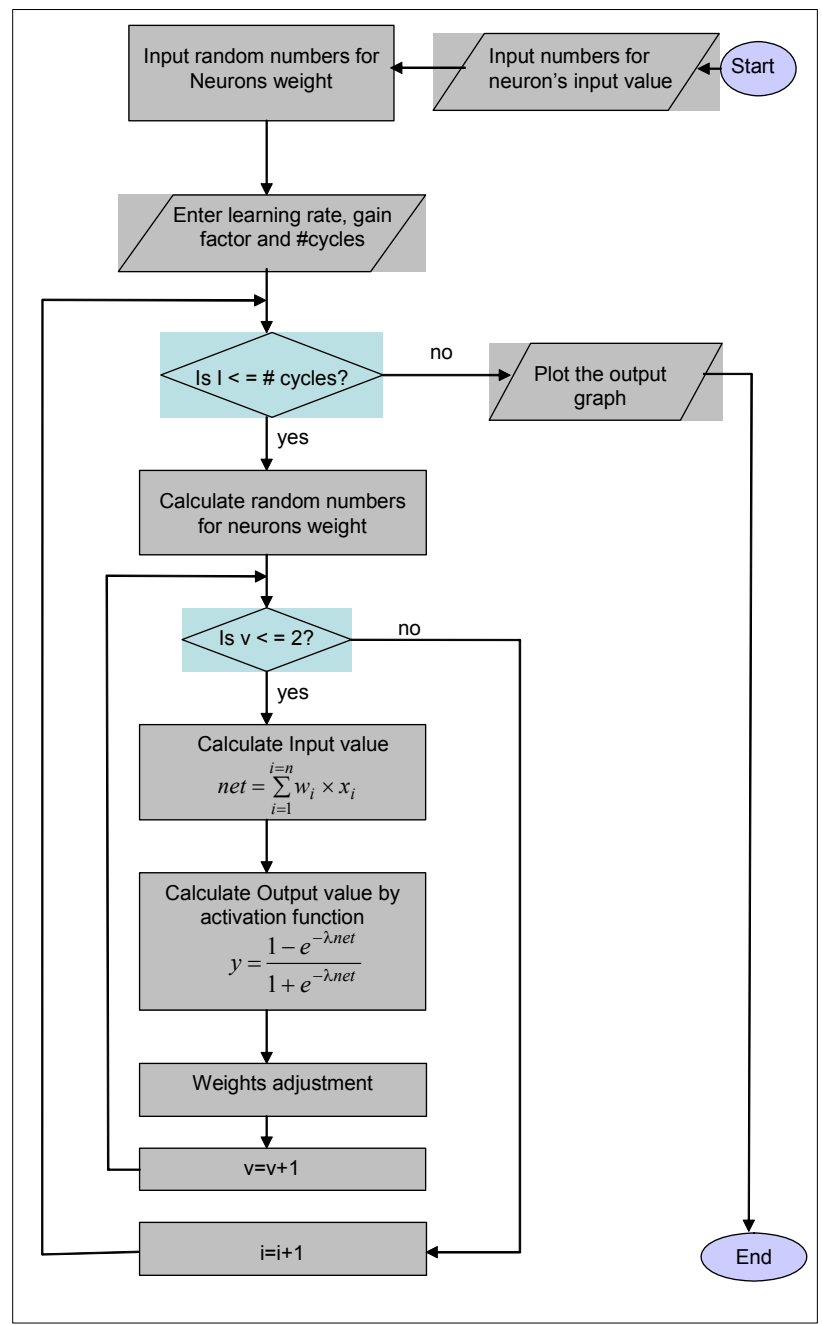

Figure 3. 